\title{
Direct Assessment of Protection Operation and Non-Viable Transients
}

\author{
Chaman Singh and Ian A. Hiskens, Senior Member, IEEE
}

\begin{abstract}
The transients induced in power systems by a large disturbance can initiate unexpected events such as protection operation or converter misfiring. This paper proposes a direct technique for assessing the likelihood of such events. The approach is based on Lyapunov (energy) function methods, but with the critical energy redefined to take account of protection operating characteristics and viability constraints. Power systems have many protection devices and many constraints that must be monitored. The paper provides a method of identifying the subset of protection devices and/or viability constraints that are most vulnerable for any particular disturbance. Controlling UEP (unstable equilibrium point) ideas underlie the algorithm.
\end{abstract}

Index Terms-Lyapunov (energy) functions, power system protection, power system security assessment, viability constraints.

\section{INTRODUCTION}

$\mathbf{V}$ OLTAGES and currents often undergo significant excursions during the transient period following the clearing of a large disturbance. This can result in unexpected switching events, which frequently have a detrimental effect on system recovery. Examples include,

- voltage collapse, where cascaded protection operation is often a contributing factor;

- large rotor swings between generators, which can result in distance protection tripping feeders, possibly leading to system separation and islanding;

- voltage dips, which may cause motors to stall, contactors to open, and power electronic converters to lose synchronism.

Therefore, a need exists for techniques that offer a fast and reliable way of, 1) assessing whether a disturbance will initiate unexpected protection and viability-related events, and 2) tuning protection and control schemes to alleviate such undesirable events.

Aspects of this have been addressed previously. The concept of a relay margin was proposed in [1] for providing a measure of the closeness of a relay to issuing a trip command. This margin was monitored along the full post-fault system trajectory to assess system vulnerability. Energy function concepts were used

Manuscript received October 18, 1999. This work was supported by the Australian Research Council through the project grant "Analysis and Assessment of Voltage Collapse," and the EPRI/DOD Complex Interactive Networks/Systems Initiative.

C. Singh is with the National Electricity Market Management Company, Carlingford, Australia.

I. A. Hiskens is with the Department of Electrical and Computer Engineering, University of Illinois at Urbana-Champaign, USA.

Publisher Item Identifier S 0885-8950(01)06060-6. in [2] for assessing out-of-step protection operation. This work was based on a heuristic (though fairly accurate) relationship between the maximum angular deviation of a separating group of generators and the minimum apparent impedance seen by out-of-step relays. The ideas were later adapted in [3] to assess voltage dip. Underlying this extension was another heuristic relationship, between maximum angular deviation and maximum voltage dip. A more general framework for Lyapunov (energy) function assessment of constrained systems was given in [4]. We have built on that general framework to rigorously incorporate protection operation and viability constraints into direct stability assessment.

Throughout this paper we will focus largely on protection operation, as it is a more intuitive concept than viability. However, the ideas extend naturally to viability concerns such as voltage sags.

Traditionally Lyapunov (energy) function techniques have been used for assessing whether a disturbance would result in generator instability [5], [6]. More recently, those techniques have been extended to load instability scenarios [7]. In general these tools have been applied to models which exhibit smooth behavior; an exception being the modeling of generator and SVC reactive power limits [8]. In all cases though, assessment of system behavior is based on a comparison of the energy acquired during the disturbance with a critical value of energy. This critical energy provides an estimate of the boundary of the stability region.

Modifications are required to include protection operation and viability limits in direct security analysis. Rather than the critical energy reflecting the stability boundary, it must now provide an estimate of the conditions which induce protection operation or nonviable transients. The first step in determining this critical energy is to establish protection and viability surfaces. A protection surface is defined as the set of points in state space at which a protection device operates. Viability surfaces are similarly defined. The critical energy is then the amount of energy that could be acquired by the system such that the post-fault trajectory just touched only one protection or viability surface, i.e., the trajectory was tangential to the surface at the point of contact. Acquisition of a larger amount of energy would result in the system trajectory crossing the surface, and hence in protection operation or violation of a viability limit.

In [9], [10], we proposed an approach to finding the critical energy based on minimizing the potential energy over all protection and viability surfaces. Unfortunately, the minimization problem is difficult to solve due to its (generally) nonconvex nature, especially for systems containing a large number of relays and constraints. In such cases it becomes almost impossible to 
incorporate all the protection and viability surfaces into the minimization process. It is necessary to reduce the complexity of the critical energy calculation by reducing the number of relays and constraints participating in the minimization.

For a particular disturbance, only a subset of all protection relays and viability limits are likely to play a role. Other relays and limits are not relevant to that disturbance, and so should not be included in calculating the critical energy. The former will be called relevant surfaces, whilst the relay or limit which is encountered first is the controlling surface. In this paper we propose a number of methods of identifying relevant surfaces and the controlling surface.

The organization of the paper is as follows. System, protection and viability models are outlined in Section II. In Section III, the importance of viability in security analysis is discussed. A method for determining the critical energy is also provided. Section IV presents methods of identifying relevant surfaces. The examples of Section V illustrate concepts developed throughout the paper. Conclusions are given in Section VI.

\section{MODELS}

\section{A. System Model}

During the development of direct protection assessment concepts, it has been convenient to use a strict Lyapunov function. Consequently, the power system model must satisfy assumptions of the form: classical machine models, constant active power loads, voltage dependent reactive power loads, and lossless system [5]. The exact details of the model can be found in many places, including [7]. In general terms, the model has a differential-algebraic (DA) structure

$$
\begin{aligned}
\dot{x} & =f(x, y) \\
0 & =g(x, y)
\end{aligned}
$$

where the dynamic states $x$ are:

- generator angles, $\delta_{i}, i=n_{0}+1, \ldots, n$,

- angular velocities, $\omega_{i}, i=1, \ldots, m$,

and the algebraic states $y$ are:

- bus voltage magnitudes, $v_{i}, i=1, \ldots, n_{0}$,

- bus voltage angles, $\delta_{i}, 1=1, \ldots, n_{0}$.

We shall assume throughout that the Jacobian $\partial g / \partial y$ is nonsingular, i.e., solutions of $g=0$ are well defined.

The corresponding Lyapunov function is

$$
\mathcal{V}(\omega, v, \delta)=\mathcal{V}_{K E}(\omega)+\mathcal{V}_{P E}(v, \delta)
$$

where

$$
\begin{aligned}
\mathcal{V}_{K E}(\omega)= & \frac{1}{2} \sum_{i=1}^{m-1} M \omega_{i}^{2} \\
\mathcal{V}_{P E}(v, \delta)= & -\frac{1}{2} \sum_{i=1}^{n} \sum_{j=1}^{n} B_{i j}\left(v_{i} v_{j} \cos \delta_{i j}-v_{i}^{s} v_{j}^{s} \cos \delta_{i j}^{s}\right) \\
& -\int_{\delta^{s}}^{\delta} P^{0^{t}} d \delta+\sum_{i=1}^{n_{0}} \int_{v_{i}^{s}}^{v_{i}} \frac{Q_{d_{i}}\left(z_{i}\right)}{z_{i}} d z_{i} .
\end{aligned}
$$

For practical applications, the modeling assumptions may be relaxed. However numerical approximation of path dependent integrals is then required [5], [6].

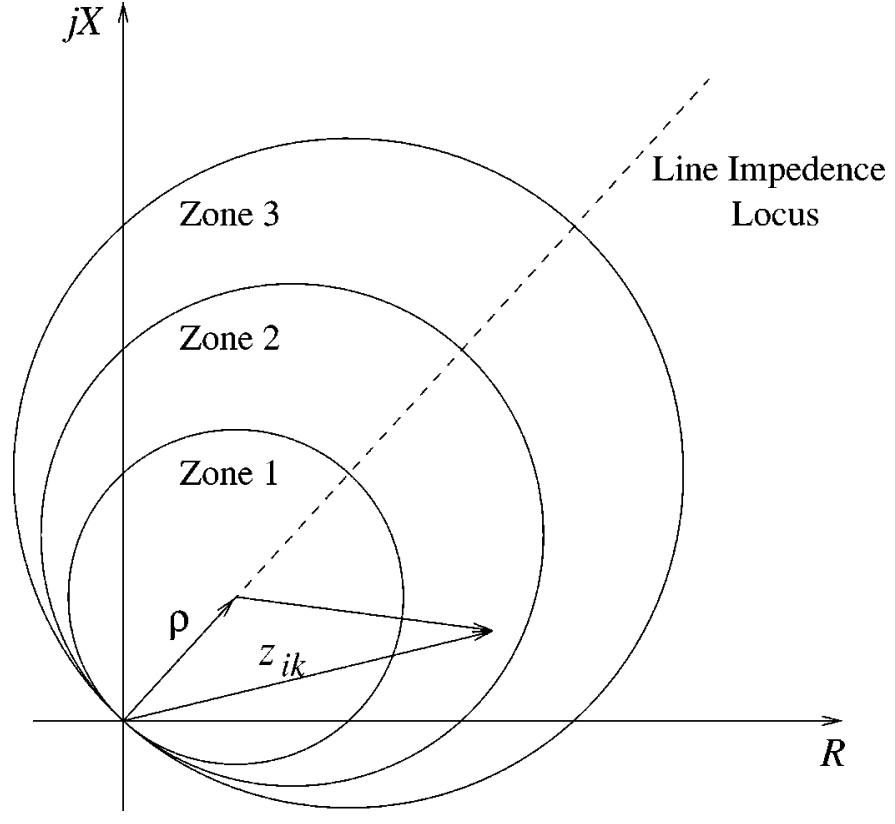

Fig. 1. Operating criteria for distance protection.

\section{B. Protection Models and Viability Constraints}

Power systems involve many different types of protection devices [11]. Examples include differential, distance and overcurrent protection. The operating characteristics of such schemes can generally be modeled as an inequality

$$
H(x, y) \leq 0 .
$$

This is illustrated in the Appendix, where distance protection is considered. Note that incorporation of out-of-step relay characteristics [11] has not been presented here, but can be handled in the same way as any other relay operating characteristic. Also time delays associated with protection devices, for example the time-coordination delays of zone 2 and 3 distance protection, have not been modeled. ${ }^{1}$

System viability constraints can also be modeled in the general form (6). For example nonviable voltages can be trivially represented as

$$
H\left(v_{i}\right)=v_{i}-v_{l} \leq 0
$$

where $v_{l}$ is a lower bound on acceptable voltage magnitudes. As mentioned earlier, transient voltage excursions below $v_{l}$ can result in motors stalling and other undesirable behavior [3].

Each protection operating region, or nonviable region, is defined by a set

$$
S_{i}=\left\{(x, y) \mid H_{i}(x, y) \leq 0\right\} .
$$

The boundary $\bar{S}_{i}$, defined by $H_{i}(x, y)=0$, forms the corresponding protection or viability surface.

As an example, each of the mho circles in Fig. 1 can be mapped into state space using such a relationship. A state space view of all the zone 1 protection relay characteristics for the

${ }^{1}$ This is not a major limitation though. A system is considered vulnerable whenever protection detects a fault. The assessment technique identifies disturbances which place the system in a vulnerable state, even if tripping is not initiated immediately. 


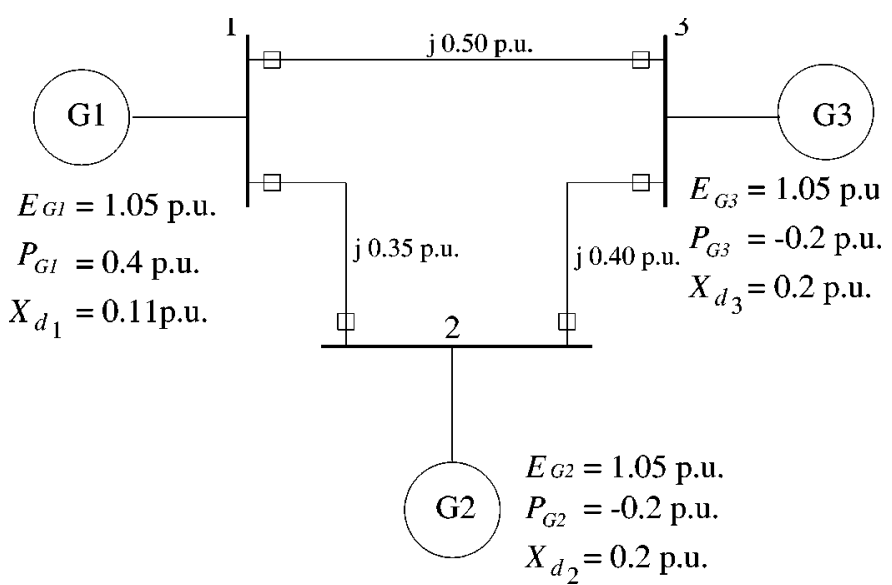

Fig. 2. Three bus example power system.

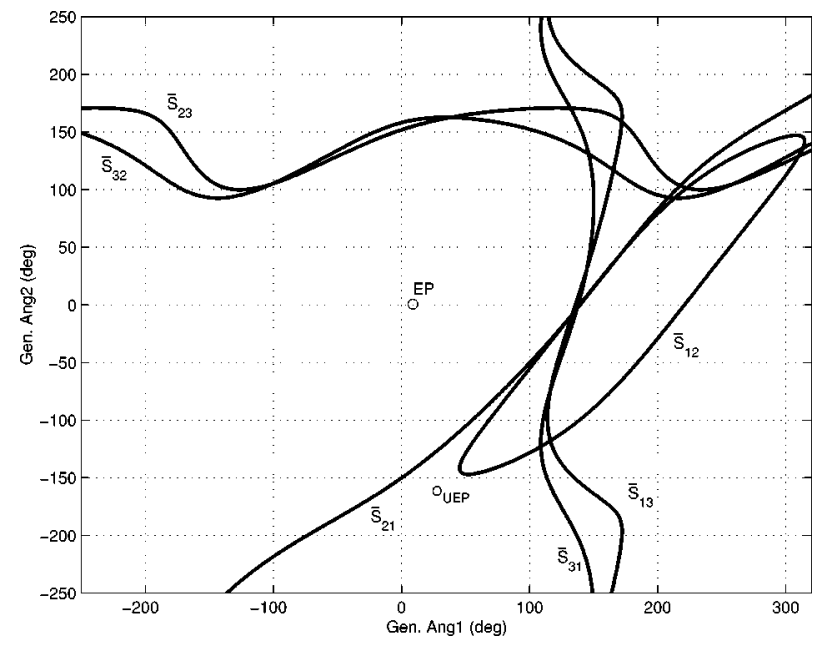

Fig. 3. State space view of protection surfaces.

system of Fig. 2 is shown in Fig. 3. Each surface $\bar{S}_{i j}$ of the figure refers to the zone 1 characteristic of the distance protection at bus $i$ on line $i-j$. These surfaces together form the boundary of the viable region. Transients should be constrained within that region to prevent unplanned switching events.

It is clear that in general the sets $S_{i}$ are quite nonconvex. This poses difficulties in solving the minimization associated with finding the critical energy [10].

\section{Stability Versus Viability}

A simple illustration using the three machine undamped power system of Fig. 2 highlights the importance of taking protection operation and viability into account in security analysis. A three phase ground fault at bus 2 was simulated. Using the controlling UEP (unstable equilibrium point) method [12], [13] it was found that the critical clearing time and critical energy were $t_{c r}=0.417 \mathrm{~s}$ and $\mathcal{V}_{u e p}=4.15$, respectively. The critically cleared system trajectory, estimated using the controlling UEP, is shown in Fig. 4. It is clear that the system is stable when protection is ignored. However the trajectory encounters a number of protection surfaces, indicating that feeders would actually be tripped.

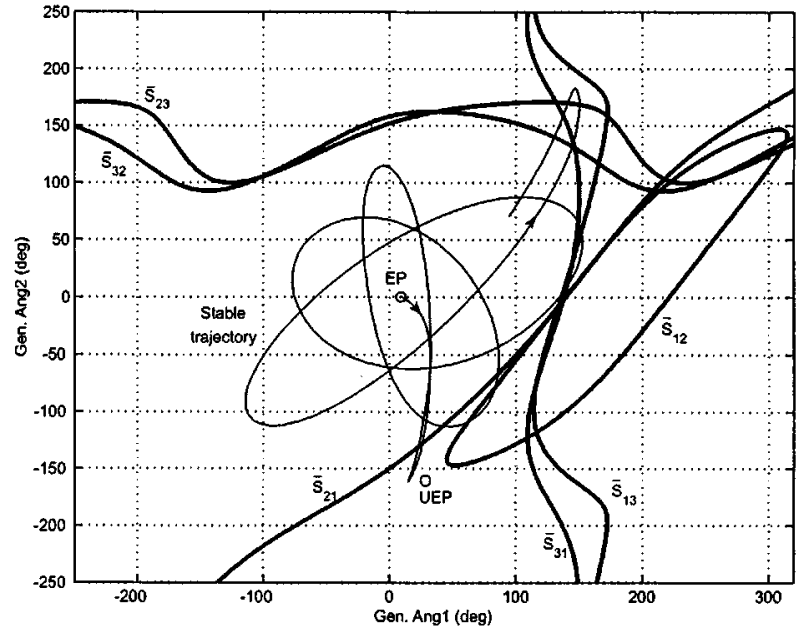

Fig. 4. Critically cleared trajectory, based on controlling UEP.

To take protection action into consideration, the critical energy should be the minimum amount of energy that could be acquired by the system such that the post-fault trajectory was tangential to a protection surface. An estimate of this critical energy can be obtained by solving the minimization

$$
\begin{gathered}
\mathcal{V}_{c r}=\min _{(x, y) \in S} \mathcal{V}_{P E}(x, y) \\
\text { s.t. } g(x, y)=0
\end{gathered}
$$

where

$$
S=\bigcup S_{i}
$$

$\mathcal{V}_{P E}(x, y)$ is given by (5), and $S_{i}$ is given by (7).

As mentioned earlier, the above optimization problem is difficult to solve due to the nonconvex nature of the protection surfaces. However a number of algorithms have been developed. Simulated annealing formed the basis of the approach reported in [10]. Reliable convergence to the global minimum was obtained, but the method was too slow for power systems of reasonable size. A gradient-based technique was developed in [14]. It was shown that robust convergence could be obtained with a predictor-corrector continuation algorithm. Computation time was also acceptable. ${ }^{2}$ The gradient-based algorithm was applied for each individual protection surface in the example. Results are given in Table I.

From Table I, the lowest potential energy occurred on $\bar{S}_{31}$, corresponding to the relay on line 3-1 at bus 3 . The critical energy was $\mathcal{V}_{c r}=3.28$, giving an estimated critical clearing time of $t_{c r}=0.373 \mathrm{~s}$. Simulation was used to check these results. The critical trajectory is shown in Fig. 5. It turned out that due to the nature of the disturbance (a fault at bus 2), $\bar{S}_{21}$ was actually encountered first, i.e., was the critical relay. The critical values given by simulation were $t_{c r}=0.403 \mathrm{~s}$ and $\mathcal{V}_{c r, s i m}=3.86$, which corresponded to a 7\% error in the estimate of $t_{c r}$.

This result illustrates that the nature of the disturbance should be taken into account when determining the critical energy $\mathcal{V}_{c r}$.

\footnotetext{
${ }^{2}$ Initial location of the minimum $\mathcal{V}_{P E}$ for each surface required around five steps, with each step computationally equivalent to a power flow. In an operational setting, a tracking mode could be used to efficiently update minima.
} 
TABLE I

MinimiZATION RESULTS (ANGLES ARE REFERRED TO G3)

\begin{tabular}{c|c|c|c}
\hline Surface & $\begin{array}{c}\text { Gen.Ang 1 } \\
\text { (deg) }\end{array}$ & $\begin{array}{c}\text { Gen.Ang 2 } \\
\text { (deg) }\end{array}$ & $\begin{array}{c}\text { Potential } \\
\text { Energy, } V_{P E}\end{array}$ \\
\hline $\bar{S}_{12}$ & 120.4 & -26.5 & 3.65 \\
\hline $\bar{S}_{13}$ & 150.7 & 53.0 & 3.46 \\
\hline $\bar{S}_{21}$ & 98.3 & -52.2 & 3.60 \\
\hline $\bar{S}_{23}$ & 174.6 & 156.4 & 3.76 \\
\hline $\bar{S}_{31}$ & 150.0 & 96.5 & 3.28 \\
\hline $\bar{S}_{32}$ & 150.8 & 142.7 & 3.55 \\
\hline
\end{tabular}

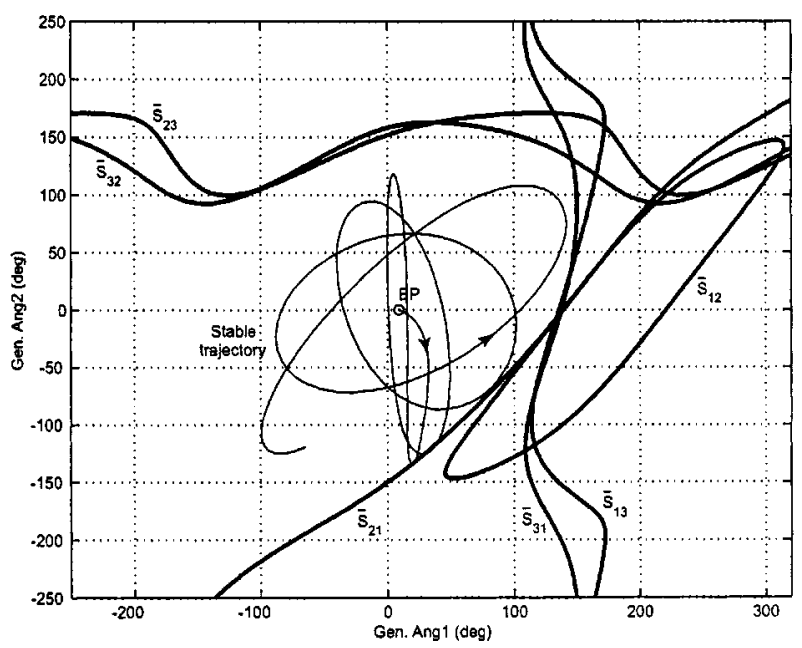

Fig. 5. Critically cleared trajectory, based on protection operation

The disturbance influences the direction of the trajectory. Only protection and viability surfaces which lie in that direction, i.e., that can possibly be intersected by the trajectory, should be considered. Otherwise the minimum $\mathcal{V}_{c r}$ might correspond to a surface which can never be encountered (for that disturbance). Use of that overall minimum $\mathcal{V}_{c r}$, as in the example, would give overly conservative stability estimates.

This concept of restricting attention to "relevant" protection and viability surfaces is similar to controlling UEP ideas. In the UEP case, the critical energy associated with a particular disturbance is given by the UEP which is relevant to that disturbance [12], [13]. In Section IV, we propose an approach for determining the relevant surfaces. The approach is motivated by controlling UEP ideas.

If the controlling protection surface $\bar{S}_{21}$ had been used in the example, the estimated critical clearing time would be $0.398 \mathrm{~s}$. This compares well with the actual value of 0.403 s given by simulation. The slight conservativeness is inherent in Lyapunov stability region estimation, and occurred because $\mathcal{V}_{K E}>0$ when the system trajectory touched the protection surface $\bar{S}_{21}$.

In Fig. 5 the system trajectory was tangential to the protection surface $\bar{S}_{21}$. If the fault clearing time $t_{c l}$ was increased slightly, the zone 1 distance protection relay at bus 2 would operate and trip line 2-1 instantaneously. Simulation with $t_{c l}=0.404$ s, i.e., just beyond the critical clearing time, is shown in Fig. 6. The system is unstable due to this subsequent protection operation.

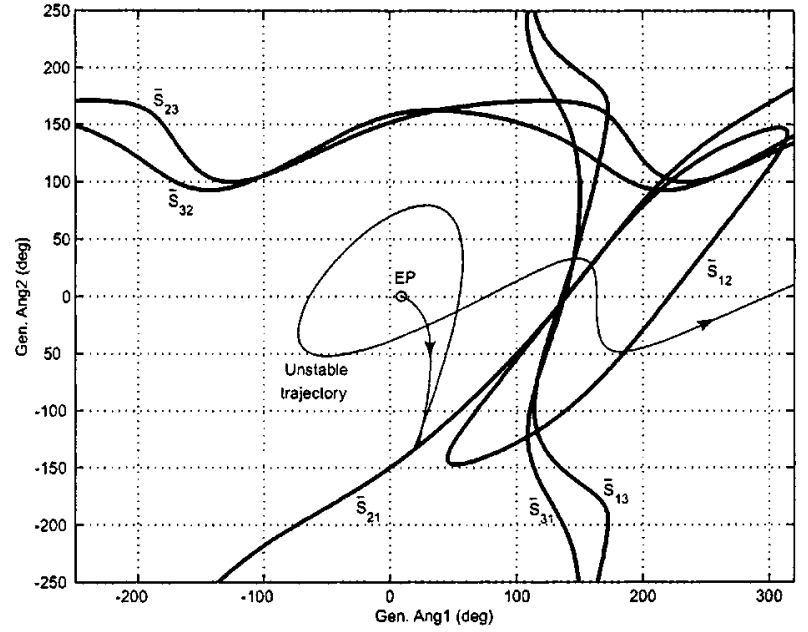

Fig. 6. Line 2-1 tripped by protection operation.

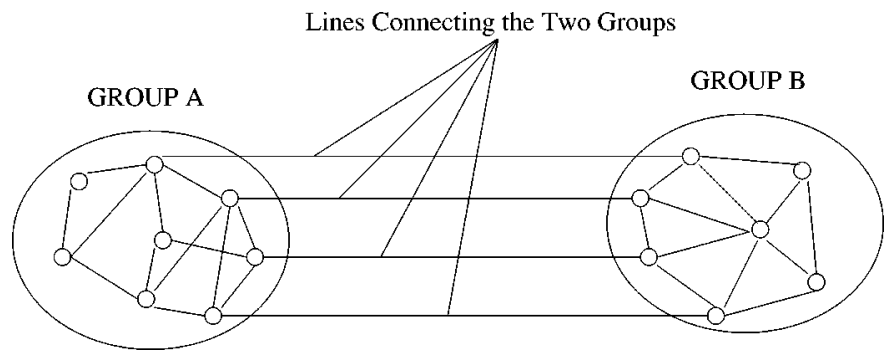

Fig. 7. Connecting network representation.

\section{Determining ReleVAnt SuRfaces}

\section{A. Justification}

Not all protection relays and viability constraints are influential following a disturbance. (In fact, generally very few will be.) Reasons for that include remoteness from the disturbance, and the nature of the transients initiated by the disturbance. Fig. 3 provides a useful illustration. It can be seen that the direction of the trajectory has quite a bearing on which relays may potentially be activated. Also, some protection characteristics cannot be reached without first encountering one of the other characteristics. Therefore, many relays and viability constraints can be eliminated from the search for the one that gives the true (disturbance dependent) $\mathcal{V}_{c r}$.

A clever approach to identifying a set of vulnerable relays was proposed in [1]. Relay ranking was based on properties of the network admittance matrix, but did not take account of the disturbance. However the previous example indicated a need for the disturbance to be considered in determining the critical energy. A procedure which achieves that aim is now proposed.

\section{B. Relevant Surface Method}

A controlling constraint method is proposed for eliminating irrelevant relays and viability limits. It is based on the properties of the controlling UEP [13], and connecting network ideas proposed in [15], where the concept of energy of separation was used.

Following a large disturbance, a power system will usually split into two coherent groups of generators, for example groups A and B of Fig. 7. The controlling UEP can be directly 
associated with that mode of instability. The network between the two groups is called the connecting network. As the two groups swing apart, i.e., the angle between the groups increases, the voltages at buses in the connecting network fall, and the angular differences across lines of the connecting network increase. Therefore viability constraints associated with buses and lines of the connecting network are relevant for the disturbance. Further, it can be seen from (16) that $w_{i k}$ (derived from the apparent impedance) will fall for lines in the connecting network. Therefore those lines become more vulnerable to protection operation.

Note though that within each coherent group, angular differences remain close to normal. So bus voltages and line angles within those groups do not deviate greatly. Consequently $w_{i k}$ remains high for those lines, and they are not susceptible to protection operation. Hence it is sensible to restrict the search for critical relays and viability constraints to lines and buses that form part of the connecting network associated with the controlling UEP.

\section{Individual Line Energy}

Dissection of the network energy into individual line energies helps to identify vulnerable lines. Expressions for line energies follow directly from the Lyapunov function (3)-(5). Kinetic energy plays no role in the line energy; only the potential energy needs to be considered. Each term of (5) can be written on a line basis as follows.

Term 1: For each line, the energy associated with the first term of (5) is simply

$$
\begin{aligned}
P E_{1, i j}= & -B_{i j}\left(v_{i} v_{j} \cos \delta_{i j}-v_{i}^{s} v_{j}^{s} \cos \delta_{i j}^{s}\right) \\
& +\frac{1}{2} B_{i j}\left(v_{i}^{2}+v_{j}^{2}-v_{i}^{s^{2}}-v_{j}^{s^{2}}\right)
\end{aligned}
$$

Term 2: It is shown in [15] that the second term can be expressed in the form of $l$ individual line energies as

$$
\int_{\delta^{s}}^{\delta} P^{0^{t}} d \delta=\sum_{k=1}^{l} \int_{\sigma_{k}^{s}}^{\sigma_{k}} p_{k}^{s} d \sigma_{k}=\sum_{k=1}^{l} P E_{2, l}
$$

where $\sigma_{k}=\delta_{i}-\delta_{j}$ is the line angle for the $k$ th line, which joins buses $i$ and $j$, and $p_{k}^{s}=B_{i j} v_{i}^{s} v_{j}^{s} \sin \sigma_{k}^{s}$ is the line power at the stable EP.

Term 3: To express the third term as line energy, it is convenient to use the reactive load model $Q_{d}(v)=Q_{0} v^{\eta}$. Then the integrals can be evaluated, giving the expression

$$
\begin{aligned}
P E_{3} & =\sum_{i=1}^{n_{0}} \int_{v_{i}^{s}}^{v_{i}} \frac{Q_{d_{i}}\left(z_{i}\right)}{z_{i}} d z_{i} \\
& =\sum_{i=1}^{n_{0}} \int_{v_{i}^{s}}^{v_{i}} Q_{0 i} z_{i}^{\eta_{i}-1} d z_{i} \\
& =\sum_{i=1}^{n_{0}} \frac{Q_{0 i}}{\eta_{i}}\left(v_{i}^{\eta_{i}}-v_{i}^{s^{\eta_{i}}}\right) \\
& =\sum_{i=1}^{n_{0}} \frac{Q_{d i}\left(v_{i}\right)-Q_{d i}\left(v_{i}^{s}\right)}{\eta_{i}}
\end{aligned}
$$

$$
=\sum_{i=1}^{n_{0}} \frac{1}{\eta_{i}}\left(\sum_{j=1}^{n} B_{i j}\left(v_{i} v_{j} \cos \delta_{i j}-v_{i}^{s} v_{j}^{s} \cos \delta_{i j}^{s}\right)\right) .
$$

The energy associated with each line can be identified from this summation in the same way as for term 1, giving

$$
\begin{aligned}
P E_{3, i j}= & \left(\frac{1}{\eta_{i}}+\frac{1}{\eta_{j}}\right) B_{i j}\left(v_{i} v_{j} \cos \delta_{i j}-v_{i}^{s} v_{j}^{s} \cos \delta_{i j}^{s}\right) \\
& -B_{i j}\left(\frac{1}{\eta_{i}}\left(v_{i}^{2}-v_{i}^{s^{2}}\right)+\frac{1}{\eta_{j}}\left(v_{j}^{2}-v_{j}^{s^{2}}\right)\right) .
\end{aligned}
$$

\section{Remarks:}

- Interesting cancellation occurs between the terms (9) and (11) for constant admittance loads, $\eta=2$.

- The form of term (11) is not applicable for constant reactive power loads, $\eta=0$. Similar analysis yields the appropriate form.

- The ZIP model for reactive load is a special case of the more general model used to derive (11).

Hence, the total energy associated with each line can be obtained as

$$
\mathcal{V}_{L, i j}=P E_{1, i j}+P E_{2, l}+P E_{3, i j}
$$

where line $l$ joins buses $i$ and $j$. As mentioned earlier, the connecting network experiences more significant bus voltage and line angle deviations than the coherent groups. Therefore $\mathcal{V}_{L, i j}$ will be greater for lines in the connecting network.

\section{Algorithm}

For a particular disturbance scenario, well established techniques can be used to solve for the controlling UEP [12], [13]. At the controlling UEP, line energies can be calculated using (12) and the $w_{i k}$ (a measure of the apparent impedance seen by relays) obtained from (16). Those values are used ${ }^{3}$ to identify line protection devices that are most vulnerable, i.e., most likely to operate. Quantities that are at risk of violating viability constraints, e.g., buses that are vulnerable to voltage dip, can be identified directly from abnormal UEP values. The examples of Section V illustrate this process.

\section{EXAMPLES}

\section{A. Three Bus Case}

The algorithm for predicting the most vulnerable relays was applied to the three bus system of Fig. 2. Faults were applied at each generator bus in turn. Results are summarized in Table II.

Table II gives the values of $\mathcal{V}_{L}$ and $w$ (based on $\beta=0.8$, zone 1) calculated at the UEP. For comparison, it also gives values of $w$ obtained from simulation. These latter values are the minimum of each $w_{i j}$ over the post-disturbance trajectory. (The fault clearing time for each case is identified in the table.) Low values of $w$ indicate vulnerable lines. High values of $\mathcal{V}_{L}$ identify lines that make a significant contribution to the critical energy $\mathcal{V}_{c r}$, and hence are vulnerable. The table shows complete correlation is all cases. For example, when bus 1 was faulted, the

\footnotetext{
${ }^{3}$ Both measures provide qualitatively similar results, so either could be used. The computational costs are small though, so we have chosen to use both to minimize the risk of overlooking vulnerable lines.
} 
TABLE II

LINE ASSESSMENT FOR THREE Bus CASE

\begin{tabular}{|c|c|c|c|c|}
\hline $\begin{array}{c}\text { Faulted } \\
\text { Bus }\end{array}$ & Line & $\begin{array}{c}V_{L} \\
\text { (uep) }\end{array}$ & $\begin{array}{c}|w| \\
(\text { uep })\end{array}$ & $\begin{array}{r}|w| \\
(\operatorname{sim})\end{array}$ \\
\hline \multirow{6}{*}{$\begin{array}{c}1 \\
\left(t_{c l}=0.285 \mathrm{~s}\right)\end{array}$} & $1-2$ & \multirow[b]{2}{*}{2.04} & 0.24 & 1.08 \\
\hline & $2-1$ & & 0.28 & 1.10 \\
\hline & $1-3$ & \multirow[b]{2}{*}{1.64} & 0.18 & 0.94 \\
\hline & $3-1$ & & 0.32 & 0.82 \\
\hline & $2-3$ & \multirow{2}{*}{0.01} & 14.44 & 1.34 \\
\hline & $3-2$ & & 14.82 & 1.16 \\
\hline \multirow{6}{*}{$\begin{array}{c}2 \\
\left(t_{c l}=0.403 \mathrm{~s}\right)\end{array}$} & $\overline{1-2}$ & \multirow[b]{2}{*}{1.77} & $\overline{00.86}$ & 1.06 \\
\hline & $2-1$ & & 0.46 & 0.80 \\
\hline & $1-3$ & \multirow{2}{*}{0.10} & 5.52 & 5.70 \\
\hline & $3-1$ & & 5.40 & 5.66 \\
\hline & $2-3$ & \multirow{2}{*}{0.74} & 0.48 & 1.02 \\
\hline & $3-2$ & & 0.86 & 1.28 \\
\hline \multirow{6}{*}{$\begin{array}{c}3 \\
\left(t_{c l}=0.395 \mathrm{~s}\right)\end{array}$} & $\overline{1-2}$ & \multirow{2}{*}{.09} & $\overline{c 7.22}$ & 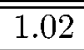 \\
\hline & $2-1$ & & 6.98 & 1.00 \\
\hline & $1-3$ & \multirow[b]{2}{*}{1.51} & 0.76 & 0.90 \\
\hline & $3-1$ & & 0.38 & 0.80 \\
\hline & $2-3$ & \multirow{2}{*}{1.02} & 0.74 & 1.16 \\
\hline & $3-2$ & & 0.40 & 0.96 \\
\hline
\end{tabular}

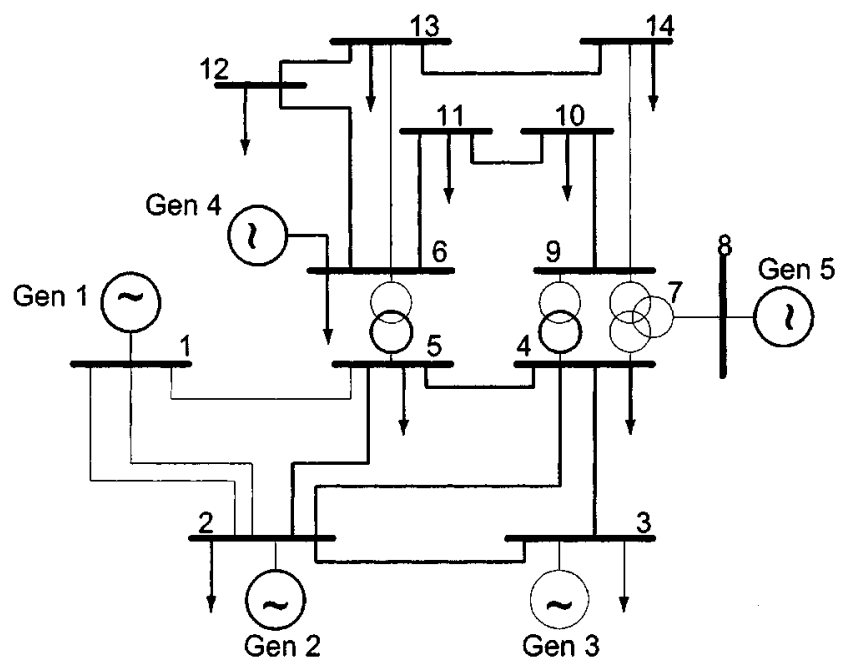

Fig. 8. IEEE 14 bus power system.

connecting network consisted of lines 1-2 and 1-3. The table shows higher values of $\mathcal{V}_{L}$ and lower values of $w$ for those lines. The other fault cases follow a similar pattern.

In Section III, a fault at bus 2 was considered. It was found that the minimum $\mathcal{V}_{c r}$ was given by line $1-3$, but the controlling surface actually corresponded to line $2-1$. It is now clear from Table II that line 1-3 is not part of the connecting network (for this disturbance), and hence is not relevant. Therefore it should not enter into the minimization (8). However line $2-1$ is part of the connecting network.

\section{B. IEEE 14 Bus Case}

The IEEE 14 bus system is shown in Fig. 8. Data for this case can be found in [16]. A three phase fault was applied at bus 3 . The critical energy of the controlling UEP was found
TABLE III

LINE ASSESSMENT FOR IEEE 14 BUS CASE

\begin{tabular}{c|c|c|c}
\hline Line & $\begin{array}{c}V_{L} \\
\text { (uep) }\end{array}$ & $\begin{array}{c}|w| \\
\text { (uep) }\end{array}$ & $\begin{array}{c}|w| \\
\text { (sim) }\end{array}$ \\
\hline $1-2$ & 0.11 & 10.66 & 5.26 \\
\hline $1-5$ & 0.02 & 11.32 & 5.52 \\
\hline $\mathbf{2 - 3}$ & \multirow{2}{*}{$\mathbf{0 . 3 8}$} & $\mathbf{0 . 9 8}$ & $\mathbf{1 . 3 0}$ \\
\cline { 1 - 3 } $\mathbf{3 - 2}$ & $\mathbf{2 . 0 2}$ & $\mathbf{2 . 1 6}$ \\
\hline $2-4$ & 0.00 & 16.50 & 15.42 \\
\hline $2-5$ & 0.00 & 36.14 & 13.56 \\
\hline $\mathbf{3 - 4}$ & \multirow{2}{*}{$\mathbf{0 . 4 6}$} & $\mathbf{2 . 0 4}$ & $\mathbf{2 . 2 0}$ \\
\cline { 1 - 4 } $\mathbf{4 - 3}$ & $\mathbf{1 . 0 6}$ & $\mathbf{1 . 4 0}$ \\
\hline $4-5$ & 0.00 & 23.36 & 24.46 \\
\hline $4-7$ & 0.02 & 7.14 & 6.64 \\
\hline $4-9$ & 0.00 & 7.28 & 6.70 \\
\hline $5-6$ & 0.00 & 6.94 & 5.32 \\
\hline $6-11$ & 0.00 & 40.28 & 28.22 \\
\hline $7-8$ & 0.01 & 9.96 & 7.20 \\
\hline $7-9$ & 0.00 & 104.36 & 26.90 \\
\hline $9-10$ & 0.00 & 112.28 & 67.02 \\
\hline $9-14$ & 0.00 & 60.32 & 31.88 \\
\hline $10-11$ & 0.00 & 45.20 & 29.54 \\
\hline
\end{tabular}

to be $\mathcal{V}_{c r, \text { uep }}=2.92$, which gave a critical clearing time $t_{c r, \text { uep }}=0.194 \mathrm{~s}$. The system was stable for $t_{c r, \text { uep }}$ when protection devices were ignored. However when distance protection was considered on all lines, with $\beta=1.6$ for zone 3 , the post-disturbance transient passed through zone 3 for line 2-3 (seen from bus 2) and for line 4-3 (seen from bus 4). Therefore protection operation should not be ignored.

Table III gives the values of $\mathcal{V}_{L}$ and $w$ (based on $\beta=1.6$ ) calculated at the controlling UEP, for selected lines. Lines 2-3 and 4-3 are correctly identified as vulnerable. ${ }^{4}$ (This is confirmed by obtaining $w$ from simulation. These values are given in Table III for comparison.) Minimizing $\mathcal{V}_{P E}$ over $S=S_{23} \bigcup S_{32} \bigcup S_{34} \bigcup S_{43}$ gave $\mathcal{V}_{c r}=2.76$. The associated estimate of the critical clearing time was $t_{c r}=0.189 \mathrm{~s}$. The actual critical clearing time, which ensured no protection operated, was $t_{c l}=0.191 \mathrm{~s}$. The estimate was therefore only $1 \%$ in error.

\section{CONCLUSION}

Operation of protection devices and mal-operation of other system components during post-disturbance transients can significantly affect the ability of a power system to recover from a disturbance. A system which was otherwise stable may lose stability because of some protection or viability induced event. The paper illustrates such a case.

Power system security assessment, including calculation of dynamic ATC, must therefore take account of stability, protection operation and viability. A direct assessment technique which achieves that objective has been proposed in this paper. Further, the proposed technique provides insights that are useful

\footnotetext{
${ }^{4}$ From a design perspective, the values of $w$ at the UEP provide guidelines for setting $\beta$ 's so that the risk of unplanned protection operation is minimized.
} 


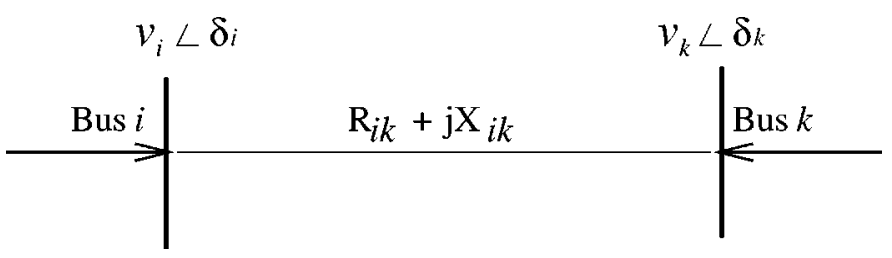

Fig. 9. Line diagram of system between buses $i$ and $k$.

for siting and tuning protection schemes such as out-of-step blocking/tripping.

Lyapunov (energy) function concepts underlie the proposed assessment technique. Energy function methods assess the security of systems by comparing the energy acquired by the system during the disturbance with a critical value. The incorporation of protection devices and viability limits requires the redefining of the critical energy. Lyapunov stability arguments have led to the critical energy being defined as the minimum potential energy over protection and viability surfaces.

For real power systems, the number of protection devices and viability constraints may be very large. The required minimization may be computationally infeasible. Therefore an approach to identifying vulnerable protection devices and relevant viability constraints has been proposed. This procedure is based on controlling UEP concepts. Its application is illustrated in the paper.

\section{APPENDIX \\ Distance PROTECTION Model}

The protection assessment concepts presented in this paper can be illustrated using distance protection [11]. Note though that the ideas extend to other forms of protection and viability limits.

Distance protection relays determine the "apparent impedance" seen from a bus, and operate if that value falls below a preset threshold. Referring to Fig. 9, the apparent impedance seen by a relay at bus $i$, along a feeder between buses $i$ and $k$ is given by

$$
z_{i k}\left(\delta_{i}, \delta_{k}, v_{i}, v_{k}\right)=\frac{v_{i} \angle \delta_{i}}{\frac{v_{i} \angle \delta_{i}-v_{k} \angle \delta_{k}}{R_{i k}+j X_{i k}}}
$$

where $v_{i}$ and $\delta_{i}$ are the voltage magnitude and angle at bus $i$, and $R_{i k}$ and $X_{i k}$ are the feeder resistance and reactance.

The operating criterion of a distance relay for a particular fault is defined according to the impedance diagram shown in Fig. 1. The relay at bus $i$ corresponding to the line between buses $i$ and $k$ will operate if

$$
\left|z_{i k}-\rho\right| \leq|\rho|
$$

where

$$
\rho=\frac{\beta}{2}\left(R_{i k}+j X_{i k}\right)
$$

is the center of the mho circle in the complex plane.

Note that each circle in Fig. 1 corresponds to a different value of $\beta$. For typical relay settings, $\beta=0.8$ for zone 1 , and $\beta=$ 1.2 for zone 2 . Zone 3 settings are power system dependent, with $\beta$ usually much higher than for zone 2 . Generally, if the system enters zone 1 , the relay will send an instantaneous trip signal. For the other zones, the system must remain within the trip region for a preset time before a trip signal will be initiated. However, the system is considered vulnerable any time these zones are encountered.

An alternate form of the operating criterion can be obtained by substituting (13) and (15) into (14), giving

$$
\begin{aligned}
& \left|\frac{v_{i} \angle \delta_{i}}{v_{i} \angle \delta_{i}-v_{k} \angle \delta_{k}}\left(R_{i k}+j X_{i k}\right)-\frac{\beta}{2}\left(R_{i k}+j X_{i k}\right)\right| \\
& \quad \leq\left|\frac{\beta}{2}\left(R_{i k}+j X_{i k}\right)\right|
\end{aligned}
$$

and hence

$$
\left|\frac{v_{i} \angle \delta_{i}}{v_{i} \angle \delta_{i}-v_{k} \angle \delta_{k}}-\frac{\beta}{2}\right| \leq \frac{\beta}{2}
$$

Letting

$$
w_{i k}\left(\delta_{i}, \delta_{k}, v_{i}, v_{k} ; \beta\right)=\left|\frac{2 v_{i} \angle \delta_{i}}{v_{i} \angle \delta_{i}-v_{k} \angle \delta_{k}}-\beta\right|
$$

gives the relay operating criterion

$$
w_{i k}\left(\delta_{i}, \delta_{k}, v_{i}, v_{k} ; \beta\right) \leq \beta .
$$

Interestingly, the actual line impedance plays no role in this expression.

The operating criterion (14) or alternatively (17) can be written in the general form

$$
H_{i k}(y) \leq 0
$$

as all load bus variables are algebraic states, i.e., elements of $y$.

\section{REFERENCES}

[1] F. Dobraca, M. A. Pai, and P. W. Sauer, "Relay margins as a tool for dynamical security analysis," International Journal of Electrical Power and Energy Systems, vol. 12, no. 4, pp. 226-234, Oct. 1990.

[2] V. Vittal, T. Oh, and A. A. Fouad, "Apparent impedance correlation of transient energy function margin and time simulation," IEEE Trans. Power Systems, vol. 3, no. 2, pp. 455-462, May 1988.

[3] A. Debs, "Voltage dip at maximum angular swing in the context of direct stability analysis," IEEE Trans. Power Systems, vol. 5, no. 4, pp. 1497-1502, Nov. 1990.

[4] K. L. Praprost and K. A. Loparo, "A stability theory for constrained dynamic systems with applications to electric power systems," IEEE Trans. Automatic Control, vol. 41, no. 11, pp. 1605-1617, Nov. 1996.

[5] M. A. Pai, Energy Function Analysis for Power System Stability. Boston, MA: Kluwer Academic Publishers, 1989.

[6] A. A. Fouad and V. Vittal, Power System Transient Stability Analysis Using the Transient Energy Function Method. Englewood Cliffs, NJ: Prentice-Hall, 1991.

[7] R. J. Davy and I.A. Hiskens, "Lyapunov functions for multi-machine power systems with dynamic loads," IEEE Trans. Circuits and Systems I, vol. 44, no. 9, pp. 796-812, Sept. 1997.

[8] I. A. Hiskens and D. J. Hill, "Incorporation of SVC's into energy function methods," IEEE Trans. Power Systems, vol. 7, no. 1, pp. 133-140, Feb. 1992.

[9] C. Singh and I. A. Hiskens, "Energy function assessment of protection operation," in Proceedings Australasian Universities Power Engineering Conference, Hobart, Australia, Sept. 1998.

[10] C. Singh, I. A. Hiskens, and P. R. Kumar, "Application of simulated annealing to power system protection assessment," in Proceedings of the 37th Conference on Decision and Control, Tampa, FL, Dec. 1998.

[11] J. L. Blackburn, Protective Relaying Principles and Applications, 2nd ed. New York: Marcel Dekker, 1998.

[12] H.-D. Chiang, F. F. Wu, and P. P. Varaiya, "A BCU method for direct analysis of power system transient stability," IEEE Trans. Power Systems, vol. 9, no. 3, pp. 1194-1200, Aug. 1994.

[13] R. T. Treinen, V. Vittal, and W. Kliemann, "An improved technique to determine the controlling unstable equilibrium point in a power system," IEEE Trans. Circuits and Systems I, vol. 43, no. 4, pp. 313-323, Apr. 1996. 
[14] C. Singh and I. A. Hiskens, "Energy function optimization for power system protection assessment," in Proceedings Australasian Universities Power Engineering Conference, Darwin, Australia, Sept. 1999.

[15] I. A. Hiskens, "Energy functions, transient stability and voltage behavior in power systems," Ph.D. dissertation, The University of Newcastle, NSW, Australia, Mar. 1990.

[16] N. Narasimhamurthi and M. T. Musavi, "A generalized energy function for transient stability analysis of power systems," IEEE Trans. Circuits and Systems, vol. 31, no. 7, pp. 637-645, July 1984.

Chaman Singh received the B.Sc. (Eng) and M.Sc. (Eng) degrees in electrical engineering from St. Petersburg State Technical University, St. Petersburg, Russia in 1994 and 1996, respectively. He received the Ph.D. degree from the University of Newcastle, Australia in 2001, and is currently with the National Electricity Market Management Company (NEMMCO)
Ian A. Hiskens (S'77-M'80-SM'96) received the B.Eng. (Elec) and B.App.Sc. (Math) degrees from the Capricornia Institute of Advanced Education, Rockhampton, Australia in 1980 and 1983, respectively. He received the Ph.D. degree from the University of Newcastle, Australia in 1990. He was with the Queensland Electricity Supply Industry from 1980 to 1992, and was a Senior Lecturer at the University of Newcastle from 1992 to 1999 . He is currently a visiting Associate Professor in the Department of Electrical and Computer Engineering at the University of Illinois at Urbana-Champaign. 